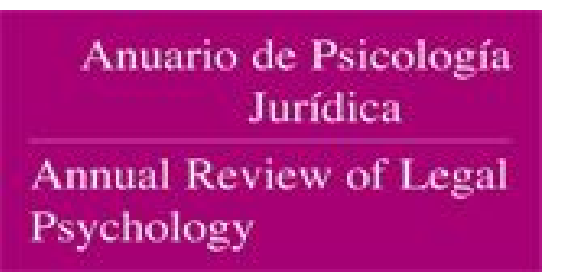
\section{Jurídica}

Annual Review of Legal Psychology

\section{Anuario de Psicología Jurídica}

ISSN: $1133-0740$

revistas_copm@cop.es

Colegio Oficial de Psicólogos de Madrid

España

Moral de la Rubia, José; Pacheco Sánchez, María Elena Consistencia Interna y Validación de la Estructura Factorial de la Escala de Conducta Disocial (ECODI27) en Tres Muestras Distintas

Anuario de Psicología Jurídica, vol. 20, 2010, pp. 15-29

Colegio Oficial de Psicólogos de Madrid

Madrid, España

Disponible en: http://www.redalyc.org/articulo.oa?id=315026299003

- Cómo citar el artículo

Número completo

- Más información del artículo

Página de la revista en redalyc.org

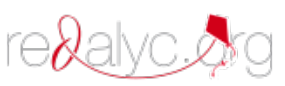

Sistema de Información Científica Red de Revistas Científicas de América Latina, el Caribe, España y Portugal Proyecto académico sin fines de lucro, desarrollado bajo la iniciativa de acceso abierto 


\title{
Consistencia Interna y Validación de la Estructura Factorial de la Escala de Conducta Disocial (ECODI27) en Tres Muestras Distintas
}

\section{Internal Consistency and Validation of the Factor Structure of the 27-item Dissocial Behavior Scale (DBS-27) in Three Different Samples}

\author{
José Moral de la Rubia \\ Universidad Autónoma de Nuevo León.
}

\author{
María Elena Pacheco Sánchez \\ Universidad Autónoma de Baja California México
}

Resumen. El estudio tenía como objetivos validar la estructura factorial de la Escala de Conducta Disocial de 27 ítems (ECODI27) en tres muestras de adolescentes: estudiantes hombres $(n=277)$, estudiantes mujeres $(n=371)$ y varones infractores $(n=158)$, determinar la consistencia interna y contrastar el ajuste de las distribuciones a una curva normal. La estructura de seis factores correlacionados presentó ajuste adecuado en las tres muestras, indicando replicabilidad. La puntuación total del ECODI27 y los factores de Robo y vandalismo y Travesuras tuvieron valores de consistencia interna altos en las tres muestras (de $0,897$ a 0,746$)$; Grafiti en mujeres $(\alpha=0,564)$ y Conducta oposicionista desafiante en infractores $(\alpha=0,568)$, los más bajos; y los restantes valores variaron de 0,887 a 0,600 . Las distribuciones de la puntuación total del ECODI27 y la mayoría de las sub-escalas mostraban normalidad en infractores, reflejando conductas normativas. Se sugiere su estudio y empleo en México y otros países hispanoparlantes.

Palabras claves: Conducta disocial, adolescentes, infractores, estudiantes de media superior, psicometría.

\begin{abstract}
The aims of the study were to validate the factor structure of the 27 -item Dissocial Behavior Scale (DBS-27) in three samples of adolescent: male students $(n=277)$, female students $(n=371)$ and male offenders $(n=158)$, to determine the internal consistency reliability and to test the fit of distributions to a normal curve. The model of correlated sixfactors showed an adequate fit within the three samples, indicating replicability. The DBS27 total score and the factors of Robbery and vandalism and Pranks had high values of internal consistency in the three samples (from .897 to .746); Graffiti in women $(\alpha=.564)$; Oppositional defiant behavior in offenders $(\alpha=.568)$ had the lowest values; and the remaining values of alpha varied from .887 to .600 . The DBS-27 total score and most of the subscales distributions were normal in the offender sample, reflecting a normative conduct. We suggested the study and use of the DBS-27 in Mexico and other Spanish-speaking countries. Key words: Dissocial behavior, adolescent, offenders, high school students, psychometrics.
\end{abstract}

La correspondencia sobre este artículo debe enviarse al primer autor a la Universidad Autónoma de Nuevo León, Facultad de Psicología, c/Mutualismo 110. Col. Mitras Centro, C.P. 64460, Monterrey, Nuevo León, México.e-mail: jose_moral@hotmail.com 


\section{Introducción}

El trastorno disocial se caracteriza por un patrón comportamental que viola los derechos básicos de los demás y reglas sociales que se espera que el niño o adolescente comprenda y respete en relación con su edad y capacidad intelectual (APA, 2000). Su prevalencia en población general adolescente es del 6 al $16 \%$ en varones y del 2 y el $9 \%$ en mujeres (APA, 2000); en población de infractores varones menores de edad, es de al menos 50\%, constituyendo el antecedente necesario para el diagnóstico de trastorno antisocial de la personalidad que está presente del 25 al 30\% de los reclusos adultos (Hare, 2003) y 3\% de la población general (APA, 2000). Así, dos tercios de los adolescentes que presentan trastorno disocial se van a acomodando a las normas y expectativas de convivencia, abandonando su patrón abusivo e irrespetuoso de comportamiento en su proceso de desarrollo.

Aunque el trastorno tiene factores de riesgo genéticos, relacionados con la desinhibición o descontrol de impulso (Alcázar-Córcoles, Bouso-Saiz y Gómez-Jarabo, 2006) y con el trastorno por déficit de atención e impulsividad (Raine, 2000), los factores ambientales toman más peso (Decuyper, De Pauw, De Fruyt, De Bolle \& De Clercq, 2009; Farrington, 2005; Silva, 2003).

La situación que vive actualmente México es un ambiente que facilita este trastorno y parece ser que se expresa de manera importante en los adolescentes, incluso en mujeres (Moral, 2005). El narcotráfico cada vez toma más presencia en la vida pública mexicana y los narcotraficantes y sicarios de los carteles de las drogas se vuelven modelos de identidad y objeto de admiración y fascinación en estudiantes de secundaria, como observan varios estudios realizados en México (Alvarado-Vázquez, 2009; Córdoba, 2002), y en la cultura popular (Héau-Lambert y Giménez, 2004). Aparte, está la corrupción y la impunidad endémica en la vida política y administrativa mexicana (Morris, 1999; Transparencia mexicana, 2007). Sin embargo debe señalarse que la representación del narcotráfico en jóvenes universitarios no es positiva y varía de la indiferencia a la satanización (Ovalle, 2005), además de existir una lucha activa del gobierno contra estas situaciones.
Dentro de este contexto, el adecuado diagnóstico del trastorno en centros educativos, el desarrollo de programas de prevención comunitarios y el tratamiento en instituciones que atienden a menores en conflicto con la ley son claves para tener una juventud y sociedad funcionales. A tal fin, se ha desarrollado en México un instrumento de evaluación para medir este trastorno, la Escala de Conducta Disocial (ECODI27), la cual puede ser empleada en los procesos de selección de alumnos, diagnóstico clínico y evaluación para su intervención.

En la muestra de 798 adolescentes, la escala ECODI27 presentó una consistencia interna alta $(\alpha \cdot=0,938)$. Con base en análisis factorial exploratorio y confirmatorio, en dicha muestra, se determinó una estructura dimensional de seis factores correlacionados: Robo y vandalismo, Travesuras, Abandono escolar, Pleitos y armas, Grafiti y Conducta oposicionista desafiante. Por análisis factorial confirmatorio, esta estructura presentó índices de ajuste de buenos (RMSSR $=0,046$, RMSEA $=0,045$, PGI $=0,956$, APGI $=0,947$, AGFI $=0,915, \mathrm{NFI}=0,904$ ) a adecuados $\left(\chi^{2} / \mathrm{g} .1=2,564, \mathrm{GFI}=0,931\right.$, NNFI $=$ $0,930, \mathrm{CFI}=0,939)$. A su vez, cinco factores alcanzaron valores altos de consistencia interna, variando de $\alpha \cdot=0,869$ (Abandono escolar) a $\alpha \cdot=0,730$ (Conducta negativista desafiante). El factor de Grafiti mostró un valor más discreto, aunque adecuado $(\alpha \cdot=0,657)$. La estabilidad temporal en un intervalo de 4 semanas en una muestra de 30 estudiantes de la escala ECODI27 fue alta $(r=0,785$, $\mathrm{p}=0,000$ ). Los índices de estabilidad temporal de sus seis factores variaron de $\mathrm{r}=0,803$ (Travesuras) a $\mathrm{r}=0,614$ (Conducta negativista desafiante) (Moral y Pacheco, 2009). La escala ECODI27 y sus 6 factores no sólo mostraban consistencia y estabilidad, sino que también bajo sesgo introducido por la deseabilidad social, medida por la escala de Sinceridad del Inventario de Personalidad de Eysenck (EPI) (Eysenck y Eysenck, 1990), siendo la correlación más alta entre el factor de Travesuras $(\mathrm{r}=0,170)$ en la muestra de 648 estudiantes. Asimismo, presentó validez criterial con el cuestionario A-D de Seisdedos y Sánchez (2001). La correlación de la puntuación total del ECODI27 con el factor de Conducta Antisocial del cuestionario A-D fue 0,536 $\mathrm{y}$ con el factor de Conducta delictiva 0,631. 
Además, demostró capacidad para diferenciar a estudiantes de infractores de las mismas edades y sexo (Pacheco y Moral, en prensa).

El presente estudio tiene como objetivos validar la estructura factorial de la escala ECODI27 en tres muestras distintas de adolescentes: estudiantes hombres, estudiantes mujeres y varones infractores; asimismo, determinar la consistencia interna, ajuste a una curva normal y descriptivos de las distribuciones, con la finalidad de mostrar la bondad del instrumento de medida y motivar su uso y estudio. El contraste del ajuste del modelo de seis factores correlacionados se realiza comparando pares de muestras, estudiantes (mujeres y hombres) y varones (estudiantes e infractores).

\section{Método}

\section{Participantes}

Se encuestó a un total de 648 alumnos, residentes en la ciudad de Mexicali y pertenecientes al nivel medio superior, los cuales cursaban los grados de segundo, cuarto y sexto semestre. Las mujeres representaban el 57\% de los estudiantes (371 de 648) y los hombres el $43 \%$ (277 de 648), siendo significativamente mayor la proporción de mujeres $\left(\chi_{(1, N=648}^{2}=13,636, p=0,000\right)$. Por otra parte, se encuestó a 148 varones infractores que se encontraban internados en el Centro de Diagnóstico de Adolescentes y el Centro de Ejecución de Medidas para Adolescentes en la ciudad de Mexicali, 54 fueron encuestados en la ciudad de Mexicali $(36,5 \%)$ y 94 en Tijuana $(63,5 \%)$.

El rango de edad varió de 15 a 18 años en las tres muestras. El promedio de edad fue mayor en infractores que estudiantes (K-W: $\chi_{(2, \mathrm{~N}=790}^{2}=9,407$, $\mathrm{p}=0,000$ ), habiendo más infractores mayores de edad. El promedio de escolaridad fue más alto en estudiantes que en infractores $\left(\mathrm{K}-\mathrm{W}: \chi_{(2, \mathrm{~N}=790}^{2}=\right.$ $16,786, p=0,000)$. La mayoría de infractores vivían con sus madres y solos, en comparación con los estudiantes quienes vivían con más frecuencia con ambos padres $\left(\chi_{(8, \mathrm{~N}=996)}^{2}=99,944, \mathrm{p}=0,000\right)$. Los padres varones de los infractores eran empleados con más frecuencia y con una carrera profesional con menos frecuencia en comparación con los padres de los estudiantes $\left(\chi_{(4, \mathrm{~N}=627)}^{2}=21,833, \mathrm{p}=0,000\right)$. Sin embargo, la distribución de frecuencias de las ocupaciones de las madres fueron estadísticamente equivalentes entre las tres muestras $\left(\chi_{(8, \mathrm{~N}=744)}^{2}=11,976, \mathrm{p}=0,152\right)$. Se halló diferencia significativa entre las tres muestras en el número de hermanos $\left(\mathrm{F}_{(2,769)}=47,312, \mathrm{p}=0,000\right)$. Las medias del número de hermanos entre los estudiantes de ambos sexos fueron equivalentes $(\mathrm{p}=0,205)$, pero diferenciales con los infractores varones $(p<0,001)$. Asimismo, hubo diferencia en el orden de hermanos $\left(\mathrm{F}_{(2,792)}=6,197, \mathrm{p}=0,002\right)$. Las medias entre los estudiantes de ambos sexos eran equivalentes en el orden de hermanos $(p=0,429)$, pero diferenciales entre estudiantes mujeres e infractores varones $(\mathrm{p}=0,002)$ y entre estudiantes hombres e infractores varones $(p=0,043)$ (véase Tabla 1 ).

\section{Instrumentos de medida}

La Escala de Conducta Disocial (ECODI27) es una escala tipo Likert de 27 reactivos con rangos de 5 puntos (de 1 totalmente de acuerdo a 5 totalmente en desacuerdo). Todos están redactados en sentido de conducta disocial. Se califican por grado de conformidad en relación con descriptores de la conducta propia. A mayor puntuación, menor presencia de conductas disociales, partiendo de los criterios del DSM-IV-TR (APA, 2000). La puntuación en la escala y sus factores se obtiene por suma simple de reactivos. Presenta una estructura de seis factores correlacionados: Robo y vandalismo $(5,8,17,18,24,25$, 26 y 27), Travesuras (6, 7, 15, 16, 20 у 21), Abandono escolar (3 y 4), Pleitos y armas (1, 2, 19, 22 y 23), Grafiti $(12,13$ y 14) y Conducta oposicionista desafiante $(9,10$ y 11) (véase Figura 1$)$.

\section{Procedimientos}

El tamaño de la muestra de estudiantes se determinó para lograr una estimación del trastorno disocial $(8,25 \%$ en hombres y mujeres) con un intervalo de confianza del $95 \%$ y un margen de error del $2 \%$ en una población de 9,656 alumnos de educación media superior en tres planteles públicos del 
Figura 1. Escala de Conducta Disocial (ECODI27)

Indique en qué grado está conforme con cada una de las siguientes afirmaciones como descriptoras de su conducta habitual

\begin{tabular}{|c|c|c|c|c|}
\hline $\begin{array}{c}\text { TA } \\
\text { Cotalmente de acuerdo }\end{array}$ & $\begin{array}{c}\text { BA } \\
\text { Bastante de acuerdo }\end{array}$ & $\begin{array}{c}\text { nAnD } \\
\text { Ni de acuerdo ni en desacuerdo }\end{array}$ & $\begin{array}{c}\text { BD } \\
\text { Bastante en desacuerdo }\end{array}$ & $\begin{array}{c}\text { TB } \\
\text { Totalmente en desacuerdo }\end{array}$ \\
\hline
\end{tabular}

Afirmaciones descriptoras de su conducta habitual

Conformidad

1. Me gusta participar en pleitos

2. Frecuentemente he tenido que pelear para defenderme

3. He pensado dejar la escuela

4. Quiero abandonar la escuela

5. Alguna vez he robado sin que nadie se dé cuenta

6. Es muy emocionante correr en auto a exceso de velocidad

7. Me gusta participar en alguna que otra travesura

8. He participado en pequeños robos sólo por experimentar qué se siente

9. No me dejo de los adultos cuando siento que no tienen la razón

10. Trato de desafiar a los profesores que han sido injustos conmigo

11. Cuando creo que los adultos no tienen la razón los desafío a que me demuestren lo contrario

12. Alguna vez he participado en graffiti con mis amigos

13. Me emociona subir a edificioes muy altos para grafitear

14. E graffiti es un arte que todos los jóvenes deben expresar

15. Cuando estoy aburrido en clase inicio algún tipo de movimiento para divertirme (ruido, tirar objetos, hacer carrilla)

16. Siento que a veces no puedo controlar mi impulso de hacer alguna travesura

17. Alguna vez le quité dinero a alguien más débil o menor que yo sólo por hacerlo

18. He participado en robos

19. Cuando me ofenden respondo inmediatamente y si es necesario hasta con golpes

20. Es divertido observar cuándo los compañeros se pelean

21. Es divertido hacer ruido y alboroto en un lugar cuando está todo en silencio

22. Es necesario andar armado porque estás expuesto a que en cualquier momento te puedan agredir

23. Cuando me agreden respondo inmediatamente con golpes

24. En algunas ocasiones me he visto involucrado en robo a casas ajenas

25. Es fácil tomar cosas ajenas porque las personas son descuidadas con sus objetos

26. Me gusta planear robos

27. He estado involucrado en actividades que dañan la propiedad ajena: autos, casas, edificios públicos y objetos

\begin{tabular}{lllll} 
TA & BA & nAnD & BD & TD \\
TA & BA & nAnD & BD & TD \\
TA & BA & nAnD & BD & TD \\
TA & BA & nAnD & BD & TD \\
TA & BA & nAnD & BD & TD \\
TA & BA & nAnD & BD & TD \\
TA & BA & nAnD & BD & TD \\
TA & BA & nAnD & BD & TD \\
TA & BA & nAnD & BD & TD \\
TA & BA & nAnD & BD & TD \\
TA & BA & nAnD & BD & TD \\
TA & BA & nAnD & BD & TD \\
TA & BA & nAnD & BD & TD \\
TA & BA & nAnD & BD & TD \\
TA & BA & nAnD & BD & TD \\
TA & BA & nAnD & BD & TD \\
TA & BA & nAnD & BD & TD \\
TA & BA & nAnD & BD & TD \\
TA & BA & nAnD & BD & TD \\
TA & BA & nAnD & BD & TD \\
TA & BA & nAnD & BD & TD \\
TA & BA & nAnD & BD & TD \\
TA & BA & nAnD & BD & TD \\
TA & BA & nAnD & BD & TD \\
TA & BA & nAnD & BD & TD \\
TA & BA & nAnD & BD & TD \\
TA & BA & nAnD & BD & TD \\
\hline
\end{tabular}

TA $\quad$ BA $n A n D \quad B D \quad T D$

TA BA nAnD BD TD

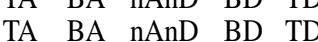

TA BA nAnD BD TD

TA $\quad$ BA $n A n D \quad$ BD $\quad$ TD

TA

TA BA nAnD BD TD

TA BA $n A n D \quad B D \quad T D$

TA BA $n A$ An $\quad$ BD $\quad$ TD

TA BA nAnD BD TD

TA $\mathrm{BA}$ nAnD $\mathrm{BD}$ TD

TA $\mathrm{BA}$ nAnD BD TD

TA BA nAnD BD TD

TA BA nAnD BD TD

TA BA nAnD BD TD

TA BA nAnD BD TD

TA BA nAnD BD TD

TA BA $n A$ AnD BD TD

TA BA nAnD BD TD

TA BA nAnD BD TD
Antes de su aplicación, el protocolo de investigación fue aprobado por el Comité de Ética de la Facultad de Medicina de la UABC y Facultad de Psicología de la UANL.

\section{Análisis estadísticos}

La consistencia interna se calculó por el coeficiente alfa de Cronbach. Valores del coeficiente $\alpha$ mayores o iguales a 0,70 se interpretan como altos, entre 0,69 y 0,60 aceptables, entre 0,59 y 0,50 bajos y menores a 0,50 inaceptables (Cervantes, 2005). El ajuste de las distribuciones a una curva normal se contrastó por la prueba de Kolmogorov-Smirnov. Se mantiene la hipótesis nula de normalidad con valores de p mayores o iguales a 0,050 y se habla de tendencia a la normalidad con valores de $p$ entre 0,049 y 0,010. Los cálculos se hicieron con SPSS16.

El ajuste de la estructura dimensional propuesta en las muestras se contrastó por análisis factorial confirmatorio, estimando la función de discrepancia por Máxima Verosimilitud (LR) y manejando todos los residuos como independientes. Los valores perdidos fueron sustituidos por las medias de sus variables para posibilitar el análisis. Se contemplaron 15 índices de ajuste: cuatro descriptivos básicos (función de discrepancia [FD], estadístico ji al cuadrado $\left[\chi^{2}\right]$, cociente entre el estadístico ji al cuadrado y sus grados de libertad $\left[\chi^{2} / \mathrm{gl}\right]$ y residuo estandarizado cuadrático medio [RMSSR]); cinco poblacionales de no centralidad (parámetro de no centralidad poblacional [PNCP], residuo cuadrático medio de aproximación [RMSEA], índice de no centralidad de McDonald [McI], índice gamma poblacional [GPI] e índice gamma poblacional ajustado [AGPI]); además se consideraron seis índices comparativos (índice de bondad de ajuste de Joreskog [GFI], índice de bondad de ajuste ajustado de Joreskog [AGFI], índice de ajuste normado de Bentler [NFI], índice comparativo de ajuste de Bentler [CFI], índice de ajuste incremental Delta2 
de Bollen [IFI] e índice Rho2 de Tucker-Lewis [TLI]). Valores de buen ajuste para los índices serían: $\mathrm{p}>0,05$ para $\chi^{2}$, cociente $\chi^{2} / \mathrm{gl}<2, \mathrm{FD}<2$, PNCP $<1$, RMSSR y RMSEA $<0,05$, AGFI, AGPI y NFI $>0,90$, así como GPI, McI, GFI, CFI, IFI y TLI $>0,95$; valores adecuados serían: $\mathrm{p}>0,01$ para ${ }^{-2}$, cociente $\chi^{2} / \mathrm{gl}<3, \mathrm{FD}<3, \mathrm{PNCP}<2$, RMSSR y RMSEA $<0,075$, AGFI, AGPI y NFI $>0,80$, así como GPI, McI, GFI, CFI, IFI y TLI > 0,85 (Moral, 2006). Los cálculos se hicieron con STATISTICA7 (tablas de parámetros conjuntos e índices descriptivos básicos y de no centralidad) y se complementaron con
AMOS7 (gráficas por muestras e índices comparativos de ajuste).

\section{Resultados}

\section{Ajuste del modelo de seis factores correlacionados en hombres y estudiantes}

En la muestra de varones infractores, todos los parámetros son significativos (véase Figura 2), así como en la muestra de mujeres estudiantes (véase

Figura 2. Modelo no constreñido estandarizado para hombres infractores

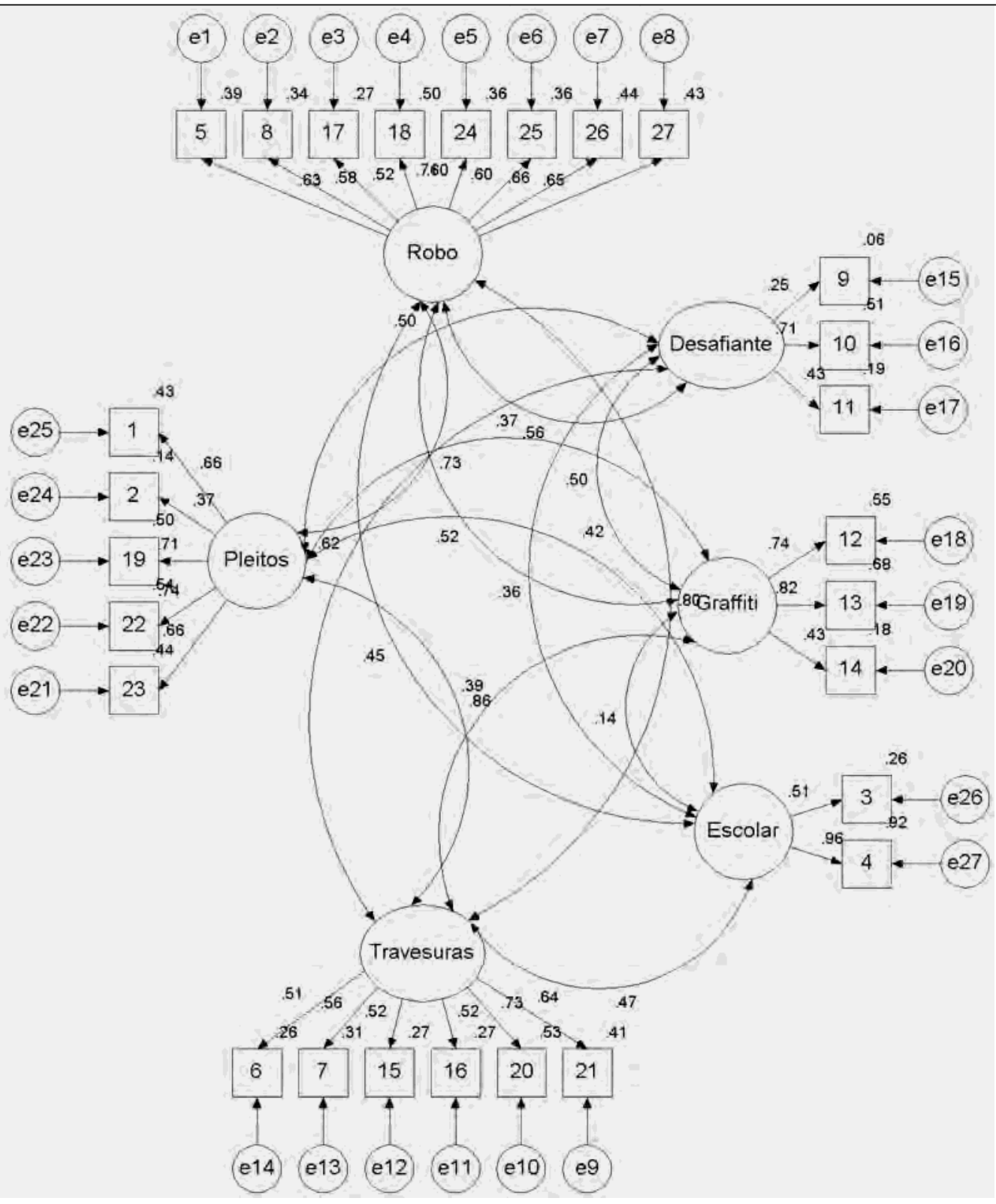


Figura 3), pero en la muestra de estudiantes varones el factor de ajuste escolar resulta independiente de conducta negativista desafiante (véase Figura 4). Todos los parámetros estimados entre varones (estudiantes e infractores) son significativos, salvo la correlación entre Ajuste escolar y Conducta negativista desafiante (véase Tabla 2). Todos los parámetros estimados entre estudiantes (mujeres y hombres) son significativos (véase Tabla 3 ).

Los índices de ajuste en las comparaciones entre estudiantes (hombres y mujeres) y varones (estudiantes e infractores) son de adecuados a buenos, aunque el modelo se rechaza por la prueba ji al cuadrado ( $p<0,001)$. El ajuste es mejor entre estudiantes (mujeres y hombres) que entre varones (estudiantes e infractores). En la comparación entre estudiantes, cuatro indicadores toman valores de buen ajuste $\left(\mathrm{FD}=1,886, \chi^{2} / \mathrm{gl}=1,846, \mathrm{PNCP}=0,864\right.$ y $\mathrm{AGPI}=$ $0,931)$ y diez alcanzan valores adecuados (RMSSR $=0,068, \quad$ RMSSEA $=0,051, \quad$ McI $=0,921$,

Tabla 2. Parámetros en las muestras de hombres estudiantes e infractores

\begin{tabular}{|c|c|c|c|c|c|c|c|c|c|}
\hline Factores & Par & E.E. & $T$ & Prob. & Residuo & Par & E.E. & $T$ & Prob. \\
\hline (Robo)-1->[R27_1] & 0,575 & 0,038 & 15,132 & 0,000 & Delta1 & 0,669 & 0,044 & 15,310 & 0,000 \\
\hline$-2->[$ R34_1] & 0,606 & 0,036 & 16,674 & 0,000 & Delta2 & 0,632 & 0,044 & 14,346 & 0,000 \\
\hline$-3->\left[R 51 \_1\right]$ & 0,495 & 0,042 & 11,817 & 0,000 & Delta3 & 0,755 & 0,041 & 18,188 & 0,000 \\
\hline$-4->\left[R 54 \_1\right]$ & 0,681 & 0,032 & 21,164 & 0,000 & Delta4 & 0,536 & 0,044 & 12,229 & 0,000 \\
\hline$-5->\left[R 63 \_1\right]$ & 0,545 & 0,040 & 13,770 & 0,000 & Delta5 & 0,703 & 0,043 & 16,328 & 0,000 \\
\hline$-6->\left[R 64 \_1\right]$ & 0,586 & 0,037 & 15,645 & 0,000 & Delta6 & 0,657 & 0,044 & 14,970 & 0,000 \\
\hline$-7->\left[R 65 \_1\right]$ & 0,595 & 0,037 & 16,115 & 0,000 & Delta7 & 0,646 & 0,044 & 14,676 & 0,000 \\
\hline$-8->\left[R 68 \_1\right]$ & 0,505 & 0,041 & 12,186 & 0,000 & Delta8 & 0,745 & 0,042 & 17,791 & 0,000 \\
\hline (Travesuras)-9-> [R29_1] & 0,549 & 0,040 & 13,790 & 0,000 & Delta9 & 0,699 & 0,044 & 16,010 & 0,000 \\
\hline$-10->\left[R 32 \_1\right]$ & 0,594 & 0,037 & 15,841 & 0,000 & Delta10 & 0,647 & 0,045 & 14,534 & 0,000 \\
\hline$-11->\left[R 46 \_1\right]$ & 0,599 & 0,037 & 16,066 & 0,000 & Delta11 & 0,642 & 0,045 & 14,392 & 0,000 \\
\hline$-12->\left[R 50 \_1\right]$ & 0,541 & 0,040 & 13,459 & 0,000 & Delta12 & 0,708 & 0,043 & 16,287 & 0,000 \\
\hline$-13->\left[R 58 \_1\right]$ & 0,674 & 0,033 & 20,347 & 0,000 & Delta13 & 0,545 & 0,045 & 12,190 & 0,000 \\
\hline$-14->\left[R 59 \_1\right]$ & 0,650 & 0,035 & 18,817 & 0,000 & Delta14 & 0,578 & 0,045 & 12,893 & 0,000 \\
\hline (Escolar)-15->[R15_1] & 0,690 & 0,051 & 13,411 & 0,000 & Delta15 & 0,523 & 0,071 & 7,367 & 0,000 \\
\hline$-16->\left[\mathrm{R} 18 \_1\right]$ & 0,956 & 0,062 & 15,396 & 0,000 & Delta16 & 0,085 & 0,119 & 0,717 & 0,474 \\
\hline (Pleitos)-17->[R6_1] & 0,651 & 0,036 & 18,300 & 0,000 & Delta17 & 0,577 & 0,046 & 12,462 & 0,000 \\
\hline$-18->\left[R 7 \_1\right]$ & 0,504 & 0,043 & 11,789 & 0,000 & Delta18 & 0,746 & 0,043 & 17,327 & 0,000 \\
\hline$-19->\left[R 55 \_1\right]$ & 0,676 & 0,034 & 19,747 & 0,000 & Delta19 & 0,543 & 0,046 & 11,717 & 0,000 \\
\hline$-20->[$ R61_1] & 0,561 & 0,040 & 13,996 & 0,000 & Delta20 & 0,685 & 0,045 & 15,247 & 0,000 \\
\hline$-21->\left[R 62 \_1\right]$ & 0,640 & 0,036 & 17,742 & 0,000 & Delta21 & 0,590 & 0,046 & 12,767 & 0,000 \\
\hline (Grafiti)-22->[R42_1] & 0,751 & 0,037 & 20,030 & 0,000 & Delta22 & 0,436 & 0,056 & 7,737 & 0,000 \\
\hline$-23->\left[R 43 \_1\right]$ & 0,702 & 0,038 & 18,275 & 0,000 & Delta23 & 0,507 & 0,054 & 9,397 & 0,000 \\
\hline$-24->\left[R 44 \_1\right]$ & 0,537 & 0,044 & 12,298 & 0,000 & Delta24 & 0,711 & 0,047 & 15,143 & 0,000 \\
\hline (Desafiante)-25-> [R37_1] & 0,561 & 0,048 & 11,622 & 0,000 & Delta25 & 0,686 & 0,054 & 12,668 & 0,000 \\
\hline$-26->\left[R 38 \_1\right]$ & 0,632 & 0,047 & 13,451 & 0,000 & Delta26 & 0,600 & 0,059 & 10,098 & 0,000 \\
\hline$-27->\left[R 40 \_1\right]$ & 0,589 & 0,048 & 12,358 & 0,000 & Delta27 & 0,653 & 0,056 & 11,632 & 0,000 \\
\hline Correlaciones entre factores & Par & E.E. & $T$ & Prob. & & & & & \\
\hline (Travesuras)-55-(Robo) & 0,520 & 0,049 & 10,644 & 0,000 & & & & & \\
\hline (Escolar)-56-(Robo) & 0,419 & 0,053 & 7,861 & 0,000 & & & & & \\
\hline (Pleitos)-57-(Robo) & 0,575 & 0,047 & 12,182 & 0,000 & & & & & \\
\hline (Grafiti)-58-(Robo) & 0,506 & 0,052 & 9,725 & 0,000 & & & & & \\
\hline (Desafiante)-59-(Robo) & 0,342 & 0,064 & 5,383 & 0,000 & & & & & \\
\hline (Escolar)-60-(Travesuras) & 0,180 & 0,057 & 3,166 & 0,002 & & & & & \\
\hline (Pleitos)-61-(Travesuras) & 0,700 & 0,041 & 16,935 & 0,000 & & & & & \\
\hline (Grafiti)-62-(Travesuras) & 0,458 & 0,055 & 8,308 & 0,000 & & & & & \\
\hline (Desafiante)-63-(Travesuras) & 0,596 & 0,055 & 10,832 & 0,000 & & & & & \\
\hline (Pleitos)-64-(Escolar) & 0,223 & 0,057 & 3,895 & 0,000 & & & & & \\
\hline (Grafiti)-65-(Escolar) & 0,222 & 0,058 & 3,800 & 0,000 & & & & & \\
\hline (Desafiante)-66-(Escolar) & 0,080 & 0,064 & 1,248 & 0,212 & & & & & \\
\hline (Grafiti)-67-(Pleitos) & 0,471 & 0,056 & 8,451 & 0,000 & & & & & \\
\hline (Desafiante)-68-(Pleitos) & 0,422 & 0,063 & 6,666 & 0,000 & & & & & \\
\hline (Desafiante)-69-(Grafiti) & 0,332 & 0,068 & 4,904 & 0,000 & & & & & \\
\hline
\end{tabular}


Figura 3. Modelo no constreñido estandarizado para hombres estudiantes

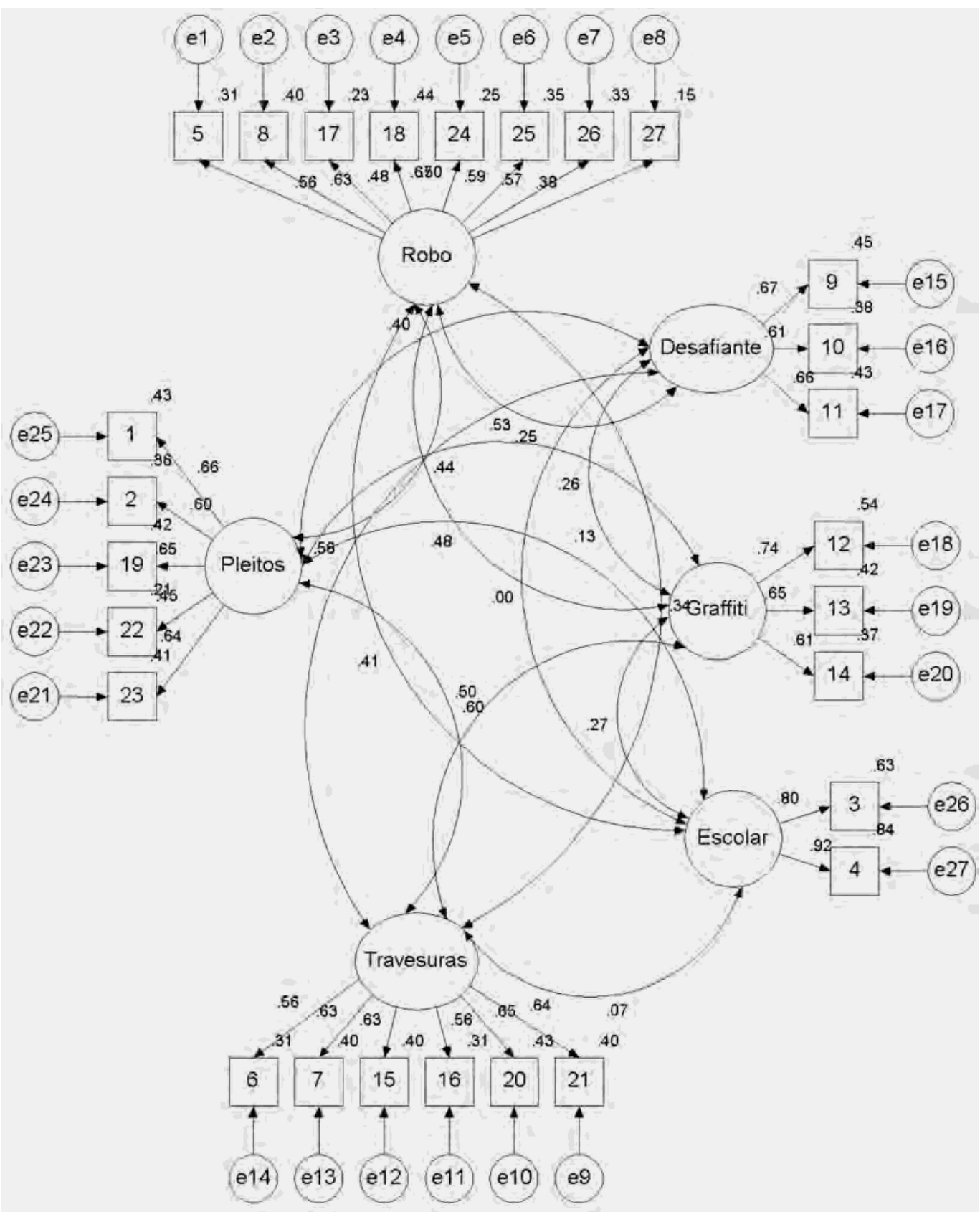

GPI $=0,940$, GFI $=0,884$, AGFI $=0,858$, NFI $=0,841$, $\mathrm{CFI}=0,890, \mathrm{IFI}=0,892$ y TLI=0,875). En la comparación entre varones, tres indicadores toman valores de buen ajuste $\left(\chi^{2} / \mathrm{gl}=1,654, \quad \mathrm{PNCP}=0,989\right.$ y AGPI $=0,922)$ y once alcanzan valores adecuados $(\mathrm{FD}=2,580, \quad \mathrm{RMSSR}=0,073, \quad \mathrm{RMSSEA}=0,055$, $\mathrm{McI}=0,872$, GPI $=0,932$, GFI $=0,861$, AGFI $=0,830$, $\mathrm{NFI}=0,849, \mathrm{CFI}=0,891, \mathrm{IFI}=0,894$ y $\mathrm{TLI}=0,879$ ) (véase Tablas 4).

\section{Consistencia interna y distribución en las tres muestras}

Los valores de consistencia interna de la escala ECODI27 (puntuación total), así como de sus factores Robo y vandalismo y Travesuras son altos en las tres muestras $(\alpha \geq 0,70)$. El factor de Abandono escolar presenta consistencia interna alta en las muestras de estudiantes y adecuada en la de infractores 
Tabla 3. Parámetros en las muestras de hombres y mujeres estudiantes

\begin{tabular}{|c|c|c|c|c|c|c|c|c|c|}
\hline Factores & Par. & $E . E$. & $T$ & Prob. & Residuo & E. E. & $T$ & Prob. & Par. \\
\hline (Robo)-1->[7_1] & 0,467 & 0,035 & 13,262 & 0,000 & Delta1 & 0,782 & 0,033 & 23,785 & 0,000 \\
\hline$-2->\left[R 34 \_1\right]$ & 0,621 & 0,029 & 21,300 & 0,000 & Delta2 & 0,615 & 0,036 & 16,997 & 0,000 \\
\hline$-3->\left[R 51 \_1\right]$ & 0,497 & 0,034 & 14,556 & 0,000 & Delta3 & 0,753 & 0,034 & 22,199 & 0,000 \\
\hline$-4->\left[R 54 \_1\right]$ & 0,675 & 0,027 & 25,295 & 0,000 & Delta4 & 0,544 & 0,036 & 15,075 & 0,000 \\
\hline$-5->\left[R 63 \_1\right]$ & 0,586 & 0,031 & 19,157 & 0,000 & Delta5 & 0,656 & 0,036 & 18,282 & 0,000 \\
\hline$-6->\left[R 64 \_1\right]$ & 0,604 & 0,030 & 20,251 & 0,000 & Delta6 & 0,635 & 0,036 & 17,596 & 0,000 \\
\hline$-7->\left[R 65 \_1\right]$ & 0,636 & 0,028 & 22,356 & 0,000 & Delta7 & 0,595 & 0,036 & 16,440 & 0,000 \\
\hline$-8->\left[R 68 \_1\right]$ & 0,491 & 0,034 & 14,301 & 0,000 & Delta8 & 0,759 & 0,034 & 22,489 & 0,000 \\
\hline (Travesuras)-9-> [R29_1] & 0,532 & 0,033 & 16,043 & 0,000 & Delta9 & 0,717 & 0,035 & 20,298 & 0,000 \\
\hline$-10->[$ R32_1] & 0,622 & 0,030 & 21,043 & 0,000 & Delta10 & 0,614 & 0,037 & 16,709 & 0,000 \\
\hline$-11->\left[R 46 \_1\right]$ & 0,591 & 0,031 & 19,171 & 0,000 & Delta11 & 0,651 & 0,036 & 17,861 & 0,000 \\
\hline$-12->\left[\mathrm{R} 50 \_1\right]$ & 0,583 & 0,031 & 18,707 & 0,000 & Delta12 & 0,660 & 0,036 & 18,177 & 0,000 \\
\hline$-13->\left[R 58 \_1\right]$ & 0,655 & 0,028 & 23,299 & 0,000 & Delta13 & 0,571 & 0,037 & 15,513 & 0,000 \\
\hline -14->[R59_1] & 0,610 & 0,030 & 20,336 & 0,000 & Delta14 & 0,627 & 0,037 & 17,124 & 0,000 \\
\hline (Escolar)-15->[R15_1] & 0,886 & 0,038 & 23,060 & 0,000 & Delta15 & 0,216 & 0,068 & 3,168 & 0,002 \\
\hline$-16->\left[\mathrm{R} 18 \_1\right]$ & 0,871 & 0,038 & 22,891 & 0,000 & Delta16 & 0,242 & 0,066 & 3,653 & 0,000 \\
\hline (Pleitos)-17->[R6_1] & 0,641 & 0,031 & 20,985 & 0,000 & Delta17 & 0,590 & 0,039 & 15,074 & 0,000 \\
\hline$-18->\left[R 7 \_1\right]$ & 0,552 & 0,034 & 16,311 & 0,000 & Delta18 & 0,695 & 0,037 & 18,606 & 0,000 \\
\hline$-19->\left[R 55 \_1\right]$ & 0,677 & 0,029 & 23,171 & 0,000 & Delta19 & 0,542 & 0,040 & 13,716 & 0,000 \\
\hline$-20->[$ R61_1] & 0,362 & 0,040 & 9,064 & 0,000 & Delta20 & 0,869 & 0,029 & 30,075 & 0,000 \\
\hline$-21->[$ R62_1] & 0,593 & 0,032 & 18,357 & 0,000 & Delta21 & 0,648 & 0,038 & 16,908 & 0,000 \\
\hline (Grafiti)-22->[R42_1] & 0,699 & 0,033 & 21,407 & 0,000 & Delta22 & 0,511 & 0,046 & 11,193 & 0,000 \\
\hline$-23->\left[R 43 \_1\right]$ & 0,651 & 0,034 & 19,418 & 0,000 & Delta23 & 0,577 & 0,044 & 13,226 & 0,000 \\
\hline$-24->\left[R 44 \_1\right]$ & 0,503 & 0,038 & 13,395 & 0,000 & Delta24 & 0,747 & 0,038 & 19,740 & 0,000 \\
\hline \multicolumn{10}{|l|}{ (Desafiante)-25-> } \\
\hline [R37_1] & 0,685 & 0,030 & 22,691 & 0,000 & Delta25 & 0,530 & 0,041 & 12,815 & 0,000 \\
\hline$-26->\left[\mathrm{R} 38 \_1\right]$ & 0,657 & 0,031 & 21,191 & 0,000 & Delta26 & 0,569 & 0,041 & 13,963 & 0,000 \\
\hline$-27->\left[R 40 \_1\right]$ & 0,718 & 0,029 & 24,443 & 0,000 & Delta27 & 0,484 & 0,042 & 11,473 & 0,000 \\
\hline Correlaciones entre factores & Par. & $E . E$. & $T$ & Prob. & & & & & \\
\hline (Travesuras)-56-(Robo) & 0,371 & 0,045 & 8,316 & 0,000 & & & & & \\
\hline (Escolar)-57-(Robo) & 0,342 & 0,042 & 8,068 & 0,000 & & & & & \\
\hline (Pleitos)-58-(Robo) & 0,380 & 0,046 & 8,276 & 0,000 & & & & & \\
\hline (Grafiti)-59-(Robo) & 0,545 & 0,043 & 12,665 & 0,000 & & & & & \\
\hline (Desafiante)-60-(Robo) & 0,199 & 0,050 & 4,008 & 0,000 & & & & & \\
\hline (Escolar)-61-(Travesuras) & 0,218 & 0,046 & 4,730 & 0,000 & & & & & \\
\hline (Pleitos)-62-(Travesuras) & 0,630 & 0,038 & 16,588 & 0,000 & & & & & \\
\hline (Grafiti)-63-(Travesuras) & 0,526 & 0,045 & 11,686 & 0,000 & & & & & \\
\hline (Desafiante)-64-(Travesuras) & 0,585 & 0,040 & 14,659 & 0,000 & & & & & \\
\hline (Pleitos)-65-(Escolar) & 0,242 & 0,047 & 5,121 & 0,000 & & & & & \\
\hline (Grafiti)-66-(Escolar) & 0,256 & 0,049 & 5,208 & 0,000 & & & & & \\
\hline (Desafiante)-67-(Escolar) & 0,139 & 0,049 & 2,855 & 0,004 & & & & & \\
\hline (Grafiti)-68-(Pleitos) & 0,531 & 0,047 & 11,393 & 0,000 & & & & & \\
\hline (Desafiante)-69-(Pleitos) & 0,511 & 0,045 & 11,454 & 0,000 & & & & & \\
\hline (Desafiante)-70-(Grafiti) & 0,295 & 0,053 & 5,545 & 0,000 & & & & & \\
\hline
\end{tabular}

$(\alpha=0,660)$. El factor de Pleitos y armas tiene consistencia interna alta en hombres y adecuada en mujeres $(\alpha=0,672)$. El factor de Grafiti muestra consistencia adecuada en la muestra de hombres $(\alpha \cdot=0,698$ en estudiantes y $:=0,694$ en infractores) y baja en la de mujeres $(\alpha=0,564)$. El factor de Conducta oposicionista desafiante cuenta con consistencia interna alta en la muestra de mujeres $(\alpha=0,752)$, adecuada en la de estudiantes hombres $(\alpha=0,681)$ y baja en la de infractores $(\alpha=0,568)$. De los 18 coeficientes $\alpha$ calculados, doce son altos, cuatro adecuados, dos bajos y ninguno resulta con un valor inaceptable (véase Tabla 5).

En la muestra de infractores, las distribuciones de la puntuación total del ECODI27 $\left(Z_{\mathrm{k}-\mathrm{s}}=0,798\right.$, $\mathrm{p}=0,547)$, de los factores de Robo y vandalismo $\left(Z_{\mathrm{k} \text {. }}\right.$ $\mathrm{s}=0,823, \quad \mathrm{p}=0,508), \quad$ Travesuras $\quad\left(\mathrm{Z}_{\mathrm{K} \cdot \mathrm{s}}=1,237\right.$, $\mathrm{p}=0,094)$, Pleitos y armas $\left(\mathrm{Z}_{\mathrm{k} \cdot \mathrm{s}}=1,333, \mathrm{p}=0,057\right)$ y 
Figura 4. Modelo no constreñido estandarizado para mujeres estudiantes

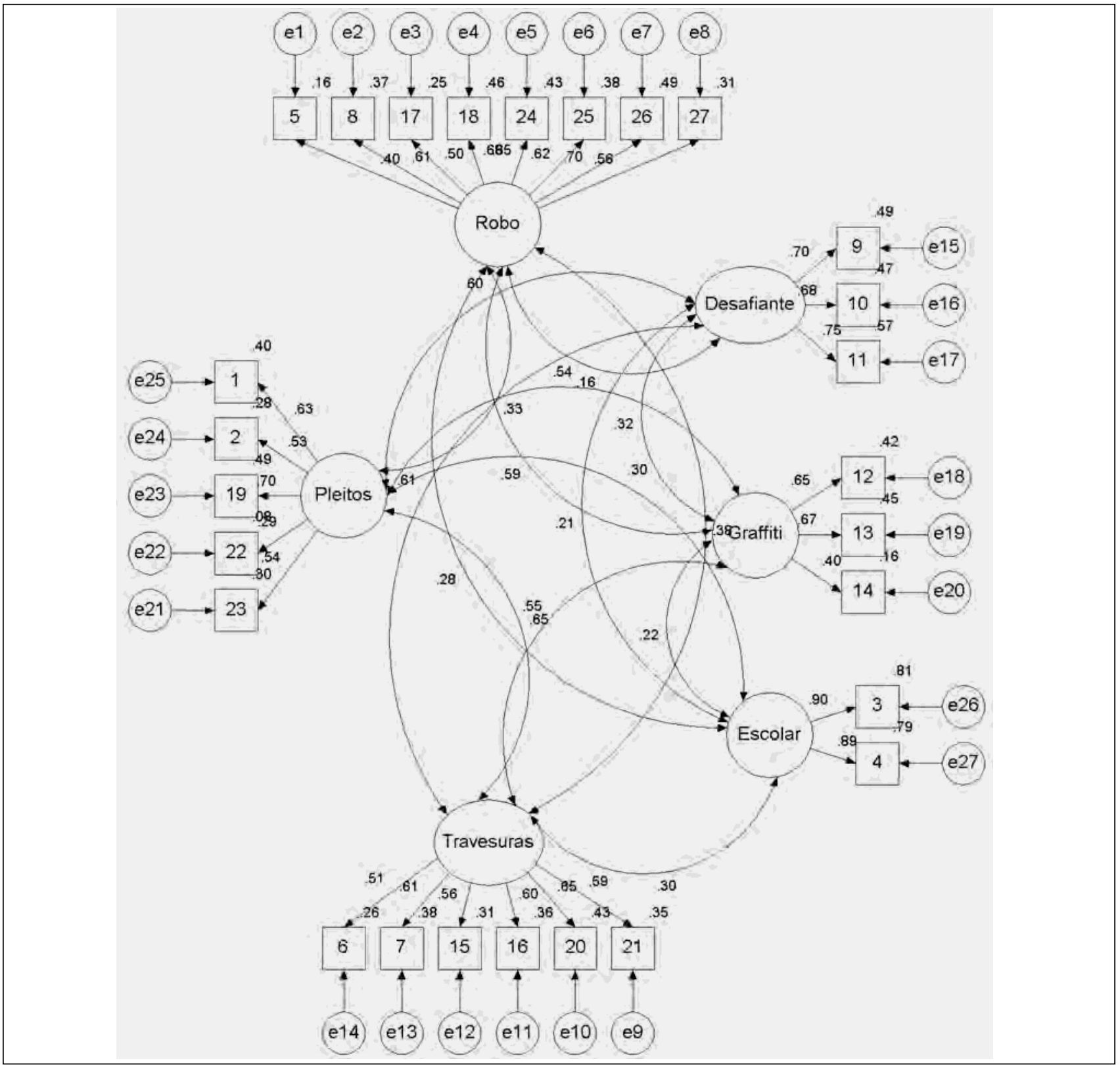

Conducta oposicionista desafiante $\left(Z_{\mathrm{K}-\mathrm{s}}=1,354\right.$, $\mathrm{p}=0,051)$ se ajustan a una curva normal, a su vez la del factor de Grafiti muestra tendencia a la normali$\operatorname{dad}\left(\mathrm{Z}_{\mathrm{K} \cdot \mathrm{s}}=1,534, \mathrm{p}=0,018\right)$. En la muestra de hombres estudiantes, las distribuciones de la puntuación total del ECODI27 $\left(Z_{\mathrm{K} . \mathrm{s}}=0,906, \mathrm{p}=0,384\right)$ y del factor de Travesuras $\left(Z_{\mathrm{K} . \mathrm{s}}=1,041, \mathrm{p}=0,229\right)$ se ajustan a una curva normal. En la muestra de mujeres, sólo la distribución del factor de Travesuras se ajusta a una curva normal $\left(\mathrm{Z}_{\mathrm{k} \cdot \mathrm{s}}=1,270, \mathrm{p}=0,080\right)$ y la distribución del factor de Conducta oposicionista desafiante se aproxima a la normalidad $\left(\mathrm{Z}_{\mathrm{k} . \mathrm{s}}=1,511, \mathrm{p}=0,021\right)$. Las demás distribuciones presentan asimetría negativa, es decir, se sesgan hacia valores por encima de la media (menor conducta disocial) (véase Tabla 5).

\section{Discusión}

El modelo de seis factores correlacionados se rechaza por la prueba ji al cuadrado, lo cual es usual en análisis factorial confirmatorio cuando se trabaja 
Tabla 4. Índices de ajuste descriptivos básicos y comparativos

\begin{tabular}{|c|c|c|}
\hline Índices de ajuste & Hombres & Estudiantes \\
\hline \multicolumn{3}{|l|}{ Índices de ajuste descriptivos básicos } \\
\hline $\begin{array}{l}\text { Función de discrepancia }(\mathrm{FD}) \\
\text { ji al cuadrado }\left(\chi^{2}\right) \\
\text { grados de libertad }(\mathrm{gl}) \\
\mathrm{p} \text { de } \chi^{2} \\
\chi^{2} / \mathrm{gl} \\
\text { RMS SR }\end{array}$ & $\begin{array}{c}2,580 \\
1091,517 \\
660 \\
0,000 \\
1,654 \\
0,073\end{array}$ & $\begin{array}{c}1,886 \\
1218,105 \\
660 \\
0,000 \\
1,846 \\
0,068\end{array}$ \\
\hline \multicolumn{3}{|l|}{ Índices de ajuste comparativos } \\
\hline $\begin{array}{l}\text { Índice de bondad de ajuste de JöresKog (GFI) } \\
\text { Índice de bondad de ajuste de JöresKog ajustado (AGFI) } \\
\text { Índice de ajuste normado de Bentler (NFI) } \\
\text { Índice comparativo de ajuste de Bentler (CFI) } \\
\text { Índice de ajuste incremental (Delta2 de Bollen) (IFI) } \\
\text { Índice de Tucker-Lewis (Rho2) (TLI) }\end{array}$ & $\begin{array}{l}0,861 \\
0,830 \\
0,849 \\
0,891 \\
0,894 \\
0,879\end{array}$ & $\begin{array}{l}0,884 \\
0,858 \\
0,841 \\
0,890 \\
0,892 \\
0,875\end{array}$ \\
\hline \multicolumn{3}{|l|}{ Índices poblacionales de no centralidad (valor medio) } \\
\hline $\begin{array}{l}\text { Parámetro de no centralidad poblacional (PNCP) } \\
\text { RMS EA } \\
\text { Índice de no centralidad de McDonald (McI) } \\
\text { Índice Gamma Poblacional (GPI) } \\
\text { Índice Gamma Poblacional Ajustado (AGPI) }\end{array}$ & $\begin{array}{l}0,989 \\
0,055 \\
0,872 \\
0,932 \\
0,922\end{array}$ & $\begin{array}{l}0,864 \\
0,051 \\
0,921 \\
0,940 \\
0,931\end{array}$ \\
\hline
\end{tabular}

con modelos grandes, como es en este caso, o muestras grandes ( $\mathrm{n}>400)$, de ahí que se acude al cociente entre el estadístico ji al cuadrado y sus grados de libertad que corrige el efecto de estas dos condiciones, especialmente la primera. Los valores del cociente menores a 2 indican buen ajuste a los datos (Moral, 2006). Considerando los valores de estos cocientes y el resto de índices de ajuste, se puede mantener el modelo de 6 factores correlacionados se ajusta de forma adecuada a los datos. Incluso se puede afirmar que la estructura factorial parece estable al replicarse en distintas muestras, ya sea de distintos sexos o poblaciones distintas del mismo sexo (estudiantes e infractores en instituciones de rehabilitación).

La falta de correlación entre Abandono escolar y Conducta oposicionista desafiante en la muestra de hombres estudiantes indica que la negativa a obedecer no conlleva abandono escolar en los varones adolescentes que estudian, aunque sí en mujeres; aun cuando el desafío oposicionista está bastante definido en el adolescente varón, ya que los promedios del factor de Conducta oposicionista desafiante son estadísticamente equivalentes entre estudiantes e infractores varones $\left(\mathrm{t}_{(423)}=1,476, \mathrm{p}=0,141\right)$, cuando son diferenciales entre mujeres estudiantes y hombres estudiantes $\left(\mathrm{t}_{(646)}=4,398, \mathrm{p}<0,001\right)$, así como entre mujeres e infractores $\left(t_{[\{1]}=5,069, p<0,001\right)$. No obstante, la distribución del desafío oposicionista por su normalidad se comporta más como rasgo en mujeres $(\mathrm{p}=0,021)$ que en hombres $(\mathrm{p}=0,001)$, además su reporte es más consistente en mujeres $(\alpha=0,752)$ que en hombres $(\alpha=0,681)$. Por lo tanto, los indicadores contemplados en el factor parecen estar funcionando mejor en mujeres que en hombres, sobre todo el referente al ítem 10 (trato de desafiar a los profesores que han sido injustos conmigo), como se ve en los parámetros de los pesos factoriales.

El factor de Grafiti se definió en el análisis factorial, cuando en un principio no se pensó en él (Moral y Pacheco, 2009). Es uno de los factores con correlaciones más altas con otros factores, sobre todo con Robos, Pleitos y Travesuras. Esto indica que la actividad de grafitear más que una expresión artística urbana es un acto antisocial, probablemente con una intención de desafío y vandalismo.

El factor de Abandono escolar cuenta con un 
Tabla 5. Consistencia interna y distribución de la escala y sus factores

\begin{tabular}{|c|c|c|c|c|c|c|c|}
\hline & ECODI27 & $F 1$ & $F 2$ & $F 3$ & $F 4$ & F5 & F6 \\
\hline Rango & $27-135$ & $8-40$ & $6-30$ & $2-10$ & $5-25$ & $3-15$ & $3-15$ \\
\hline Reactivos & 27 & 8 & 6 & 2 & 5 & 3 & 3 \\
\hline \multicolumn{8}{|c|}{ Hombres infractores $(N=148)$} \\
\hline$\alpha$ & 0,897 & 0,831 & 0,753 & 0,660 & 0,764 & 0,694 & 0,568 \\
\hline Mínimo & 31 & 8 & 6 & 2 & 5 & 3 & 3 \\
\hline Máximo & 124 & 40 & 29 & 10 & 25 & 15 & 15 \\
\hline Media & 83,422 & 26,223 & 17,811 & 7,027 & 15,162 & 9,365 & 7,831 \\
\hline D. E. & 19,632 & 7,904 & 5,334 & 2,282 & 4,928 & 3,498 & 2,825 \\
\hline Mediana & 85 & 26 & 19 & 6 & 16 & 10 & 8 \\
\hline Asimetría* & $-0,431$ & $-0,101$ & $-0,296$ & $-0,448$ & $-0,381$ & $-0,204$ & 0,411 \\
\hline Curtosis* & $-0,071$ & $-0,643$ & $-0,312$ & $-0,677$ & $-0,724$ & $-0,823$ & $-0,014$ \\
\hline $\mathrm{Z}_{\mathrm{K}-\mathrm{S}}$ & 0,798 & 0,823 & 1,237 & 2,172 & 1,333 & 1,534 & 1,354 \\
\hline$P^{\mathrm{n}-\mathrm{S}}$ & 0,547 & 0,508 & 0,094 & 0,000 & 0,057 & 0,018 & 0,051 \\
\hline \multicolumn{8}{|c|}{ Hombres estudiantes $(N=277)$} \\
\hline$\alpha$ & 0,861 & 0,746 & 0,781 & 0,844 & 0,736 & 0,698 & 0,681 \\
\hline Mínimo & 61 & 17 & 6 & 2 & 6 & 3 & 3 \\
\hline Máximo & 135 & 40 & 30 & 10 & 25 & 15 & 15 \\
\hline Media & 102,011 & 35,321 & 19,376 & 8,412 & 18,682 & 11,957 & 8,263 \\
\hline D.E. & 14,884 & 4,547 & 5,658 & 2,102 & 4,341 & 2,967 & 2,905 \\
\hline Mediana & 102 & 37 & 19 & 9 & 19 & 12 & 8 \\
\hline Asimetría* &,- 294 & $-1,195$ &,- 049 & $-1,312$ &,- 573 & $-1,024$ &, 377 \\
\hline Curtosis* &,- 275 & 1,180 &,- 605 &, 671 &,- 147 &, 485 &,- 415 \\
\hline $\mathrm{Z}_{\mathrm{K}-\mathrm{S}}$ & 0,906 & 2,526 & 1,041 & 4,308 & 1,654 & 2,590 & 2,013 \\
\hline $\mathrm{P}^{\mathrm{K}-\mathrm{S}}$ & 0,384 & 0,000 & 0,229 & 0,000 & 0,008 & 0,000 & 0,001 \\
\hline \multicolumn{8}{|c|}{ Mujeres estudiantes $(N=371)$} \\
\hline$\alpha$ & 0,858 & 0,773 & 0,759 & 0,887 & 0,672 & 0,564 & 0,752 \\
\hline Mínimo & 71 & 21 & 6 & 2 & 9 & 5 & 3 \\
\hline Máximo & 135 & 40 & 30 & 10 & 25 & 15 & 15 \\
\hline Media & 110,97 & 37,72 & 21,13 & 8,69 & 21,11 & 13,01 & 9,31 \\
\hline D. E. & 12,807 & 3,166 & 5,248 & 2,063 & 3,329 & 2,160 & 3,076 \\
\hline Mediana & 113 & 39 & 21 & 10 & 22 & 14 & 9 \\
\hline Asimetría* & $-0,564$ & $-1,843$ & $-0,407$ & $-1,630$ & $-0,983$ & $-1,082$ & $-0,143$ \\
\hline Curtosis* & $-0,107$ & 3,953 & $-0,237$ & 1,576 & 0,848 & 0,537 & $-0,662$ \\
\hline $\mathrm{Z}_{\mathrm{K}-\mathrm{S}}$ & 1,670 & 4,764 & 1,270 & 6,197 & 2,399 & 3,642 & 1,511 \\
\hline$P^{\mathrm{n}-\mathrm{s}}$ & 0,008 & 0,000 & 0,080 & 0,000 & 0,000 & 0,000 & 0,021 \\
\hline
\end{tabular}

número reducido de indicadores, sólo dos, y a pesar de ello muestra una consistencia interna alta $(\alpha>0,70)$ en las tres muestras. No obstante, se sugiere ampliar los indicadores del mismo, ensayando reactivos, como "me aburre estudiar", "veo como algo inútil ir a la escuela", "la escuela es una pérdida de tiempo" y "en la calle se aprende más cosas que en la escuela"; esto es, ítems cuyo contenido connota motivos y deseos de abandonar la escuela y no volver a la misma en caso de deserción escolar, que son consonantes con los rasgos de búsqueda de sensaciones y desinhibición conductual del trastorno.

Los rasgos disociales presentan distribuciones normales en la muestra de infractores, lo que puede interpretarse como que son normativos y adaptativos en esta población, con la excepción de Abandono escolar. La falta de normalidad de este último factor se puede atribuir al reducido número 
de reactivos que lo integran que limitan su rango, de ahí también la sugerencia anterior de ampliar los mismos. A su vez, la distribución de la puntuación total del ECODI27 en la muestra de estudiantes varones es normal, aunque con un promedio significativamente mayor que el de los infractores $\left(t_{(423)}=10,942, p<0,001\right)$; es decir, lo disocial está menos definido en estudiante. Desde esta normalidad en la distribución se puede afirmar que la conducta irrespetuosa, rebelde e irresponsable en estudiantes varones no es rasgo atípico, sino que presenta un amplio rango, no presentándose su desaprobación total en el participante promedio $(\bar{X} / \mathrm{NR}=$ $102 / 27=3,78 \approx 4=$ En desacuerdo). Sólo el factor de Travesuras muestra normalidad en todas las muestras, incluso en las mujeres, lo que puede ser un rasgo para la edad adolescente (Cava y Musitu, 2000), incluso interactuar con la cultura mexicana.

Las variables demográficas resultaron diferenciales entre estudiantes e infractores con la excepción de la ocupación de las madres, siendo la mayoría amas de casa. La falta de diferencias en la ocupación materna se podría atribuir a que el rol ocupacional es independiente de la comunicación abierta con la madre que es el factor protector de conducta disocial (Estévez, Murgui, Moreno y Musitu, 2007), y no refleja estatus social que es un factor con efecto significativo (APA, 2000; Bordallo, Zagalaz y Arenas, 1995) frente a la ocupación paterna que sí indica estatus social.

Si se intenta clasificar con las variables demográficas al conjunto de participantes, separando entre estudiantes e infractores por la técnica de regresión logística lineal, se logra un 93\% de acierto. El 98\% de los estudiantes es clasificado correctamente, así como el $62 \%$ de los infractores. El modelo de clasificación presenta ajuste significativo a los datos $\left(\chi^{2}\right.$. $\mathrm{N}=5922=3,884, \mathrm{p}=0,867)$ y explica el $67 \%$ de la varianza del criterio (ser o no infractor). Los predictores significativos del modelo son: escolaridad $(1,298 \pm$ 0,$\left.209 ; \mathrm{W}_{(1)}=38,618, \mathrm{p}<0,001 ; \mathrm{OR}=3,663\right)$, persona con la que se vive $\left(\mathrm{W}_{44}=16,769, \mathrm{p}=0,002\right)$, número de hermanos $\left(0,856 \pm 0,212 ; \mathrm{W}_{\mathrm{(1)}}=16,346, \mathrm{p}<0,001\right.$; $\mathrm{OR}=2,354)$, ocupación paterna $\left(\mathrm{W}_{22}=9,770\right.$, $\mathrm{p}=0,008) \quad \mathrm{y}$ edad $\left(-0,713 \pm 0,305 ; \quad \mathrm{W}_{(1)}=5,470\right.$, $\mathrm{p}=0,019 ; \mathrm{OR}=0,490)$. Mayor escolaridad, el vivir con ambos padres, el que padre sea profesionista, menor número de hermanos y menor edad son factores protectores, es decir, predicen ser estudiante. Menor escolaridad, el vivir con la madre o solo, el que el padre sea empleado, mayor número de hermanos y mayor edad son factores de riesgo, es decir, predicen ser infractor. Así, los datos reflejan que una familia nuclear estable y una adecuada escolaridad previenen claramente los problemas de conducta disocial; por el contrario, la desestructuración de la familia y el desfase o abandono escolar aumenta la probabilidad de conducta disocial, considerando que este modelo de clasificación es más específico (rechazo correcto del no caso) que sensible (detección del caso). Debe señalarse que estos resultados son convergentes con otros estudios, como el de García y Armas (2008) sobre estilos de crianza y Estévez et al. (2007) sobre comunicación familiar. Por otra parte, la ocupación del padre, incluso el éxito escolar, nos habla de aspectos de estructura social y diferencias de clases que no deben ser obviados en las políticas de desarrollo social para la prevención del delito.

Como limitaciones del estudio, se carece de una muestra de mujeres infractoras por la dificultad de acceso a las mismas y el hecho de que la mayoría no prestaron su consentimiento para participar en el estudio. Las diferencias demográficas y los señalamientos como factores de riesgo y protección deben interpretarse como descriptivos y no como inferenciales, para lo cual se requeriría una muestra probabilística de población general, de ahí que no se incluyó como un objetivo y sólo se presentó en la discusión de las características muestrales. Los datos que proporciona la escala ECODI27 son de auto-informe y presentan ligero sesgo introducido por la deseabilidad social. Sería interesante completar el estudio de la escala con datos de otra naturaleza, como fisiológicos, proyectivos u observacionales.

En conclusión, la estructura de seis factores correlacionados para la escala ECODI27 presenta un ajuste adecuado en las tres muestras, indicando replicabilidad. Se sugiere ampliar el número de indicadores del factor de Abandono escolar, por su reducido número e incluso para lograr la normalidad de su distribución en población de infractores. La puntuación total y los factores de Robo y vandalismo y Travesuras se muestran consistencia interna alta en 
las tres muestras. Los valores de consistencia más bajos fueron los del factor de Grafiti en mujeres y Conducta oposicionista desafiante en infractores, pero resultaron mayores a 0,50 . Los restantes valores de consistencia fueron altos $(\searrow 0,700)$ y uno en infractores, dos en mujeres y uno en hombres adecuados (entre 0,699 y 0,600 ). La puntuación total y la mayoría de las escalas mostraron distribuciones normales en infractores, reflejando un rasgo o conductas normativas. Sólo el factor de Travesuras presentó normalidad en las tres muestras. Se sugiere el estudio y empleo de la escala ECODI27 no sólo en México, sino en otros países hispanoparlantes.

\section{Referencias}

Alcázar-Córcoles, M.A., Bouso-Saiz, J.C. y GómezJarabo García, J. (2006). Estudio exploratorio sobre la caracterización del patrón desinhibido de conducta en una muestra de menores infractores de España, México y El Salvador. Anuario de Psicología Jurídica, 16, 115-137.

Alvarado-Vázquez, R.I. (2009, septiembre). La construcción de la identidad de los estudiantes de bachillerato en Sinaloa. En Consejo Mexicano de Investigación en Psicología Educativa (Ed.), Memoria electrónica del X congreso nacional de investigación educativa. Extraído el 6 de noviembre de 2009, de http://www.comie.org.mx/congreso/memoria/v10/pdf/area_tematica_16/po nencias/0172-F.pdf

American Psychiatry Association (APA) (2000). Diagnostic and Statistical Manual of Mental Disorders, 4th edition, text revision. Washington, DC: Author.

Bordallo, A., Zagalaz, M.A. y Arenas, B. (1995). Trastornos de conducta infantiles en medios socioeconómicos diferentes. Revista de la Asociación Española de Neuropsiquiatría, $X V(55), 609-625$.

Cava, M.J. y Musitu, G. (2000). Perfil de los niños con problemas de integración social en el aula. Revista de Psicología Social, 15, 319-333.

Cervantes, V. (2005). Interpretaciones del coeficiente alpha de Cronbach. Avances en Medición, 3, 928.
Córdoba, N. (2002). Narcocultura en Sinaloa: simbología, transgresión y medios de comunicación (tesis de doctorado). México: UNAM.

Decuyper, M., De Pauw, S., De Fruyt, F., De Bolle, M. \& De Clercq, B. J. (2009). A meta-analysis of psychopathy, antisocial PD- and FFM associations. European Journal of Personality, 23, 531565.

Estévez, E., Murgui, S., Moreno, D. y Musitu, G. (2007). Estilos de comunicación familiar, actitud hacia la autoridad institucional y conducta violenta del adolescente en la escuela. Psicothema, 19, 108-113.

Eysenck, H.J. y Eysenck, S.B.G. (1990). EPI (Cuestionario de Personalidad). Madrid: TEA.

Farrington, D.P. (2005). The importance of child and adolescent psychopathy. Journal of Abnormal Child Psychology, 33, 489-497.

García-Medina, P. y Armas-Vargas, E. (2008). Comorbilidad, personalidad, estilos educativos y problemas de conducta en adolescentes. Anuario de Psicología Jurídica, 18, 21-30.

Hare, R.D. (2003). Hare Psychopathy ChecklistRevised (PCL-R). Technical manual. North Tonawanda, NY: Multi-Health Systems.

Héau-Lambert, C. y Giménez, G. (2004). La representación social de la violencia en la trova popular mexicana. Revista Mexicana de Sociología, 66, 627-659.

Moral, J. (2005). Alexitimia, desviación psicopática y depresión. Un estudio en una muestra de población universitaria. Revista Medicina Universitaria, 7, 105-115.

Moral, J. (2006). Análisis factorial confirmatorio. En R. Landero y M. T. González (Ed.), Estadística con SPSS y metodología de la investigación (pp. 445-528). México: Trillas.

Moral, J. y Pacheco, M. E. (2009). Desarrollo de una Escala de Conducta Disocial en México. Enviado para su publicación.

Morris, S.D. (1999). Corruption and the Mexican political system: Continuity and change. Third World Quarterly, 20, 623-643.

Seisdedos, N. y Sánchez, P. (2001). Cuestionario de conductas antisociales y delictivas A-D. México: El Manual Moderno.

Silva, A. (2003). Conducta antisocial: un enfoque 
psicológico. México: Ed. Pax.

Ovalle, L.P. (2005). Entre la indiferencia y la satanización. Representaciones sociales del narcotráfico desde la perspectiva de los universitarios de Tijuana. Culturales, 1, 63-89.

Raine, A. (2002). Annotation: The role of prefrontal deficits, low autonomic arousal, and early health factors in the development of antisocial and aggressive behavior in children. Journal of Child Psychology and Psychiatry, 43, 417- 434.

Pacheco, M.E. y Moral, J. (en prensa). Distribución, punto de corte y validez de la Escala de Conducta Disocial (ECODI27). Revista Mexicana de Orientación Educativa (REMO).

Thrusfield, M., Ortega, C., de Blas, I., Noordhuizen, J.P. \& Frankena, K. (2001). WIN EPISCOPE 2.0: improved epidemiological software for veterinary medicine. Veterinary Record, 148, 567-572.

Transparencia mexicana (2007). Índice nacional de corrupción y buen gobierno. Extraído el 5 de octubre de 2009, de http://www.transparenciamexicana.org.mx/ENCBG/

Manuscrito recibido: 25/11/2009

Revisión recibido: 03/03/2010

Manuscrito aceptado: 12/04/2010 